\title{
Figure S2
}

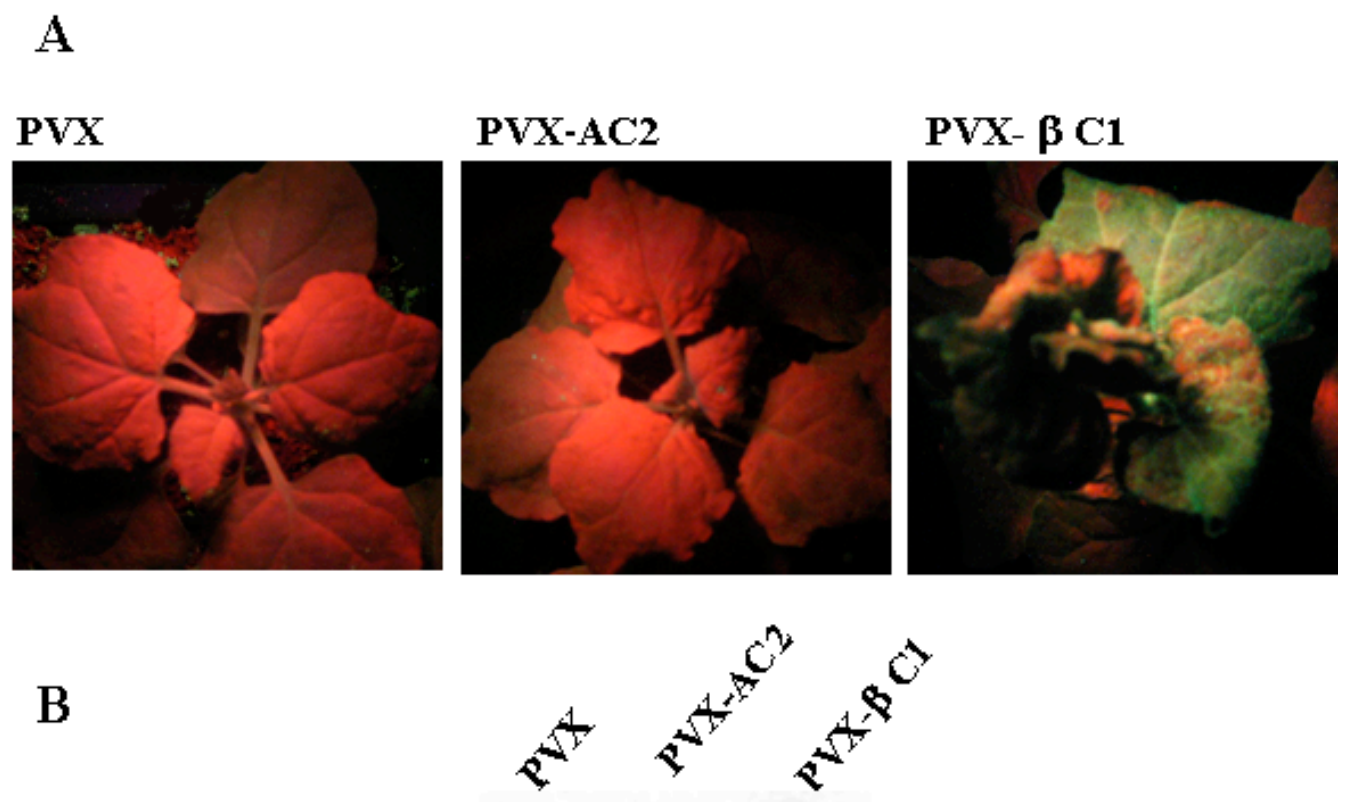

GFP mRNA

18S RRNA

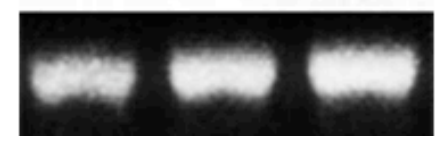

Figure S2. TYLCCNV AC2 expression cannot reverse TGS of a GFP transgene. (A) 16-TGS plants were inoculated with the PVX vector, PVX expressing AC2 from TYLCCNV (PVX-AC2), or PVX expressing $\beta C 1$ (PVX- $\beta C 1$ ), and were photographed under UV light 14 days post-inoculation. Results shown are representative of at least three independent experiments with 4 to 8 plants per treatment. (B) Gel blot analysis of RNA from leaves of 16-TGS plants inoculated as indicated. The ${ }^{32} \mathrm{P}$-labeled probe was specific for GFP mRNA. The $18 \mathrm{~S}$ rRNA loading controls were visualized by ethidium bromide staining. 